\title{
Biological intensity-modulated radiotherapy plus neoadjuvant chemotherapy for multiple peritoneal metastases of ovarian cancer: A case report
}

\author{
SHIGAO HUANG*, YAZHENG DANG* ${ }^{*}$, FUJUN LI, WEI WEI, YUXIN MA, \\ SONG QIAO and QIANYUN WANG
}

Department of Oncology, Chinese People's Liberation Army 323 Hospital, Xi'an, Shaanxi 710054, P.R. China

Received May 23, 2014; Accepted November 26, 2014

DOI: $10.3892 / 01.2014 .2820$

\begin{abstract}
The current study presents the case of a 68-year-old female patient who received biological intensity-modulated radiotherapy (BIMRT) and neoadjuvant chemotherapy for multiple peritoneal metastases of ovarian cancer. The International Federation of Gynecology and Obstetrics disease stage was IIIc. In addition, the patient presented with urination and defecation difficulties. The result of tumor marker detection showed a carcinoembryonic antigen level of $348.2 \mathrm{ng} / \mathrm{ml}$, a cancer antigen 125 level of 2,091 U/ml and a cancer antigen 19-9 level of $113 \mathrm{U} / \mathrm{ml}$. Computed tomography (CT) indicated and ovarian cystic or solid package, enlargement of multiple abdominal and retroperitoneal lymph nodes and abdominal cavity effusion. Positron emission tomography/CT indicated multiple internal organ metastases. The center of the ovarian cystic or solid package was considered to be a malignant tumor. A large amount of ascites were detected, as well as abdominal and retroperitoneal lymph node metastasis. The patient was treated with BIMRT at a total dose of $48 \mathrm{~Gy}$, administered as a single 4.0-Gy dose 12 times. In addition, $100 \mathrm{mg}$ cisplatin was administered as a peritoneal perfusion, followed by two cycles of $180 \mathrm{mg}$ Taxol and $100 \mathrm{mg}$ cisplatin. Furthermore, the enlargement of the lymph nodes was reduced and the tumor in the region of the ovary had decreased in size by $90 \%$. The ascites had disappeared and the abdominal pain was greatly improved. At the time of writing this manuscript, the patient was well and without relapse. Therefore, modern radiotherapy techniques, such as BIMRT, may be considered as a beneficial treatment option for ovarian cancer patients with multiple peritoneal metastases in whom surgery is not suitable.
\end{abstract}

Correspondence to: Mr. Shigao Huang, Department of Oncology, Chinese People's Liberation Army 323 Hospital, 6 Construction West Road, Xi'an, Shaanxi 710054, P.R. China

E-mail: huangshigao2010@aliyun.com

*Contributed equally

Key words: peritoneal multiple metastasis, ovarian cancer, radiotherapy, neoadjuvant chemotherapy

\section{Introduction}

As the fifth most common cause of cancer-related mortality, ovarian cancer accounts for $>50 \%$ of all mortalities associated with gynecological cancer (1). With an incidence of $35-78 \%$, nodal metastases occur frequently, particularly in advanced-stage ovarian tumors (stages III-IV) $(2,3)$.

Primary debulking surgery (PDS) is the current standard treatment for advanced ovarian cancer, followed by post-surgical chemotherapy (4). An improved prognosis may be expected in cases where optimal debulking (residual disease, $<1 \mathrm{~cm}$ ) can be achieved (5). Neoadjuvant chemotherapy (NAC) has been recognized as an alternative treatment to PDS for patients with a poor performance status or apparently unresectable bulky tumors (6). NAC is expected to become a standard treatment or one of the effective treatment options for advanced ovarian cancer (7) as other phase III studies $(8,9)$ begin to produce similar positive results. Radiotherapy may be an effective treatment modality, even in the setting of otherwise chemotherapy refractory disease (10). Tactics for the consolidation of a complete response following chemotherapy remain of great interest, but future studies are required to determine which consolidation treatment is optimal for advanced ovarian cancer (11).

A previous study has indicated that the majority of females with advanced ovarian cancer, in whom tumor control was achieved, will go on to develop recurrent disease (12). Combined positron emission tomography/computed tomography $(\mathrm{PET} / \mathrm{CT})$ is particularly useful for differentiating between ovarian cancer and benign disease, and for locating distant metastases. Borderline tumors may be interpreted as benign on PET/CT (13). In the present study, PET/CT technology was used to guide the use of the radiotherapy. The study was approved by the Ethics Committee of the People's Liberation Army (PLA) 323 Hospital (Xi'an, China) and the patient provided written informed consent.

\section{Case report}

This study presents the case of a 68 -year-old female who underwent biological intensity-modulated radiotherapy (BIMRT) and neoadjuvant chemotherapy for multiple peritoneal metastases of ovarian cancer [International Federation 
A

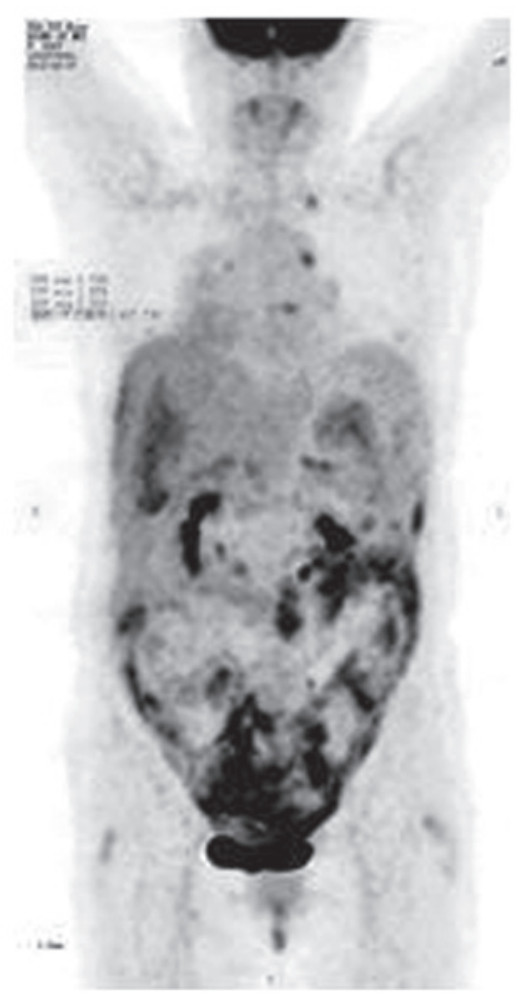

B

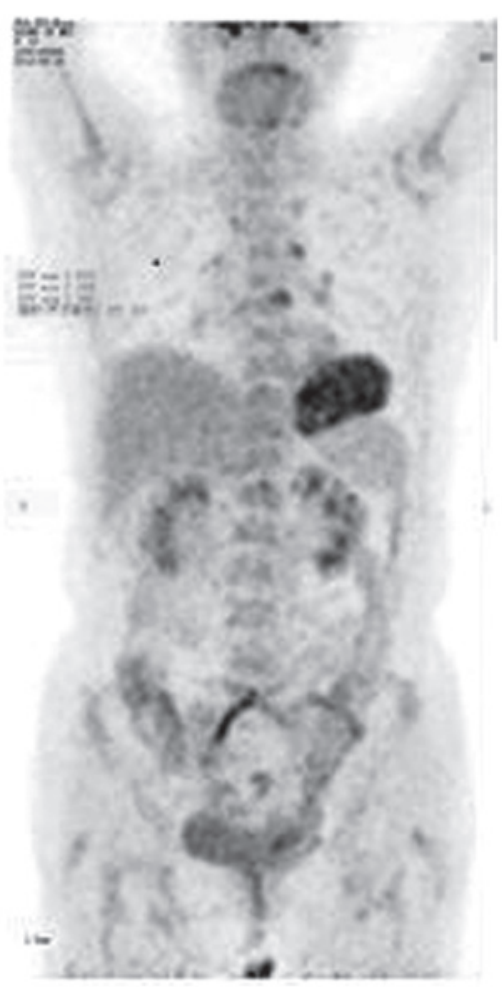

Figure 1. (A) PET revealing abnormal ${ }^{18} \mathrm{~F}$-fluorodeoxyglucose uptake in the ovary and enterocoelia, indicating a malignant ovarian tumor. (B) PET images obtained at one year post-radiotherapy treatment. PET, positron emission tomography.

of Gynecology and Obstetrics (FIGO) stage IIIc] (14) on February 2, 2012, at the PLA 323 Hospital. The patient presented with urination and defecation difficulties, and felt severe pain at multiple abdominal sites. The PET/CT examination indicated an ovarian cystic or solid package, frequent abdominal and retroperitoneal lymph node enlargement and abdominal cavity effusion (Fig. 1A and 2A). Transverse, coronary and sagittal PET/CT scans of the patient for peritoneal metastases prior to treatment revealed the presence of metastatic cancer (Fig. 3A).

Following BIMRT, two cycles of neoadjuvant combination chemotherapy (180 mg Taxol and $100 \mathrm{mg}$ cisplatin) were administered. In the Department of Oncology, radiation treatment was administered at a total dose of $48 \mathrm{~Gy}$, as single 4.0-Gy doses 12 times. A total of $100 \mathrm{mg}$ cisplatin was initially administered via peritoneal perfusion, with two cycles of chemotherapy. One chemotherapy cycle involved Taxol $\left(180 \mathrm{mg} / \mathrm{m}^{2}\right)$ dissolved in $500 \mathrm{ml}$ intravenous saline, administered intravenously over 3 hours, following a 1-hour interval, $100 \mathrm{mg} / \mathrm{m}^{2}$ cisplatin was injected. In total, two cycles, each lasting 21 days were completed. The severe pain previously experienced by the patient, particularly the abdominal pain, was alleviated by symptomatic treatment. The tumor shrank and the patient's condition was stabilized (Fig. 1B). Furthermore, PET/CT images following treatment showed normalizing ${ }^{18} \mathrm{~F}$-fluorodeoxyglucose uptake in the para-aortic lymph nodes (Figs. 2B and 3B). Following radiotherapy treatment, the CA-125 tumor marker level declined sharply, while the CA19-9 and CEA levels declined gradually. All the tumor marker levels eventually returned to within the normal ranges (Fig. 4).
At the time of writing this manuscript, the patient is well and without relapse.

\section{Discussion}

Ovarian cancer is one of the most commonly occurring gynecological malignancies Patients with ovarian carcinoma have a poor prognosis, as in the majority, the diagnosis is made at an advanced stage. Recurrence occurs frequently, particularly in the initial two years after first-line therapy. There have previously been no studies on the use of modern radiotherapy techniques, such as BIMRT, in ovarian cancer patients with multiple peritoneal metastases in whom surgery is not suitable. The majority of studies have reported the use of initial surgery or chemotherapy, followed by subsequent radiotherapy, for the treatment of ovarian cancer $(15,16)$. However, the clinical outcome remains unsatisfactory (17).

The current standard treatment of maximal cytoreductive surgery and adjuvant combination carboplatin and taxane chemotherapy is aggressive, however, the prognosis for patients with an advanced disease stage remains poor. The median time to recurrence is less than two years, and the predominance of recurrence occurs intraperitoneally. The five-year survival rate for FIGO stage IIIc disease is 20-25\% (18-20). According to the present results, it is reasonable to guide treatment based on the BIMRT for ovarian cancer patients with multiple peritoneal metastases in whom surgery is not suitable. Rochet et al (21) showed the clinical feasibility of intensity-modulated whole abdominal radiotherapy in combination with modern chemotherapy and surgery. The technique provides coverage of the entire peritoneal cavity, including frequent sites of abdominal 

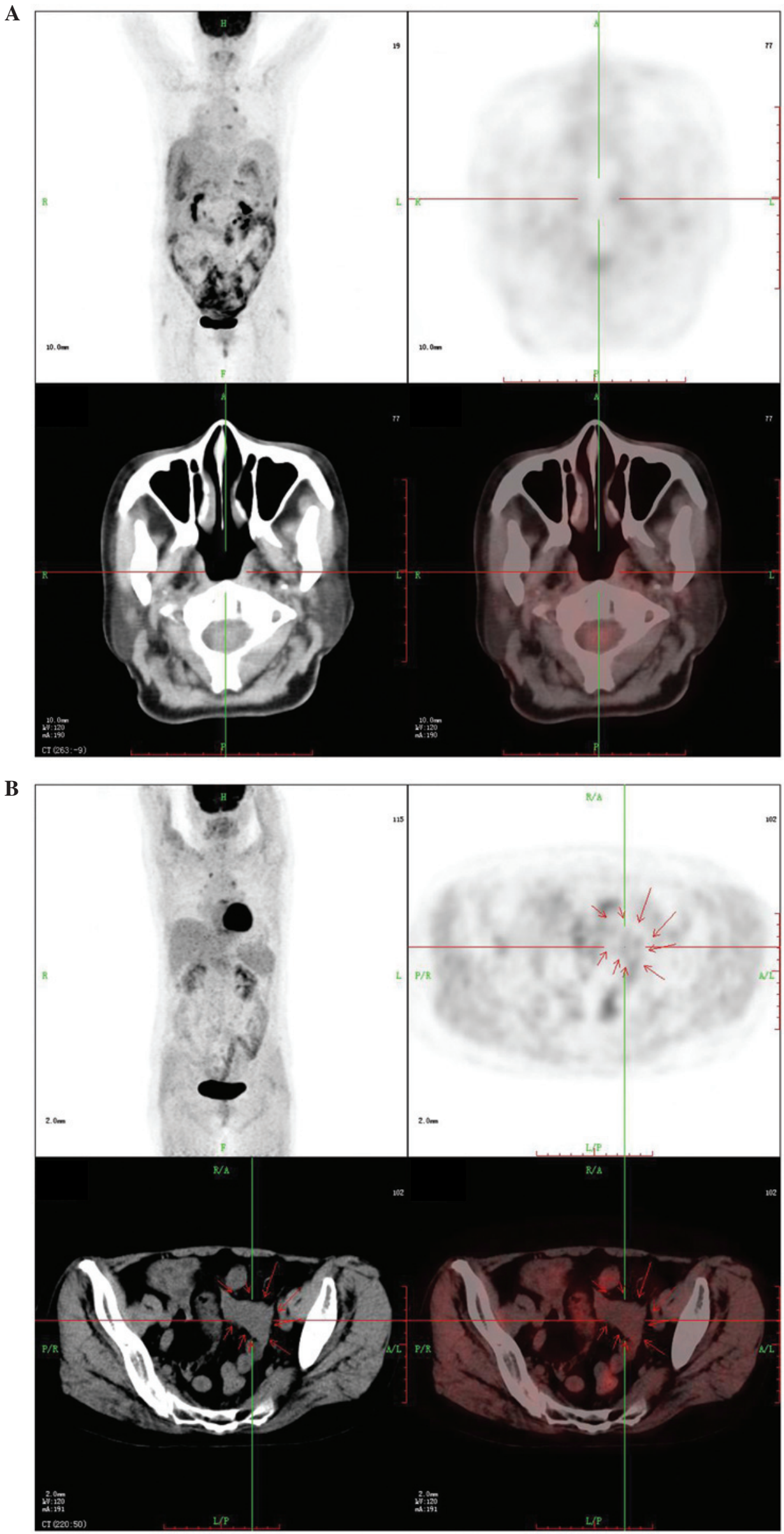

Figure 2. Positron emission tomography/computed tomography of (A) various regions prior to treatment and (B) normalizing ${ }^{18} \mathrm{~F}$-fluorodeoxyglucose uptake in the para-aortic lymph nodes of the patient following radiotherapy treatment. 

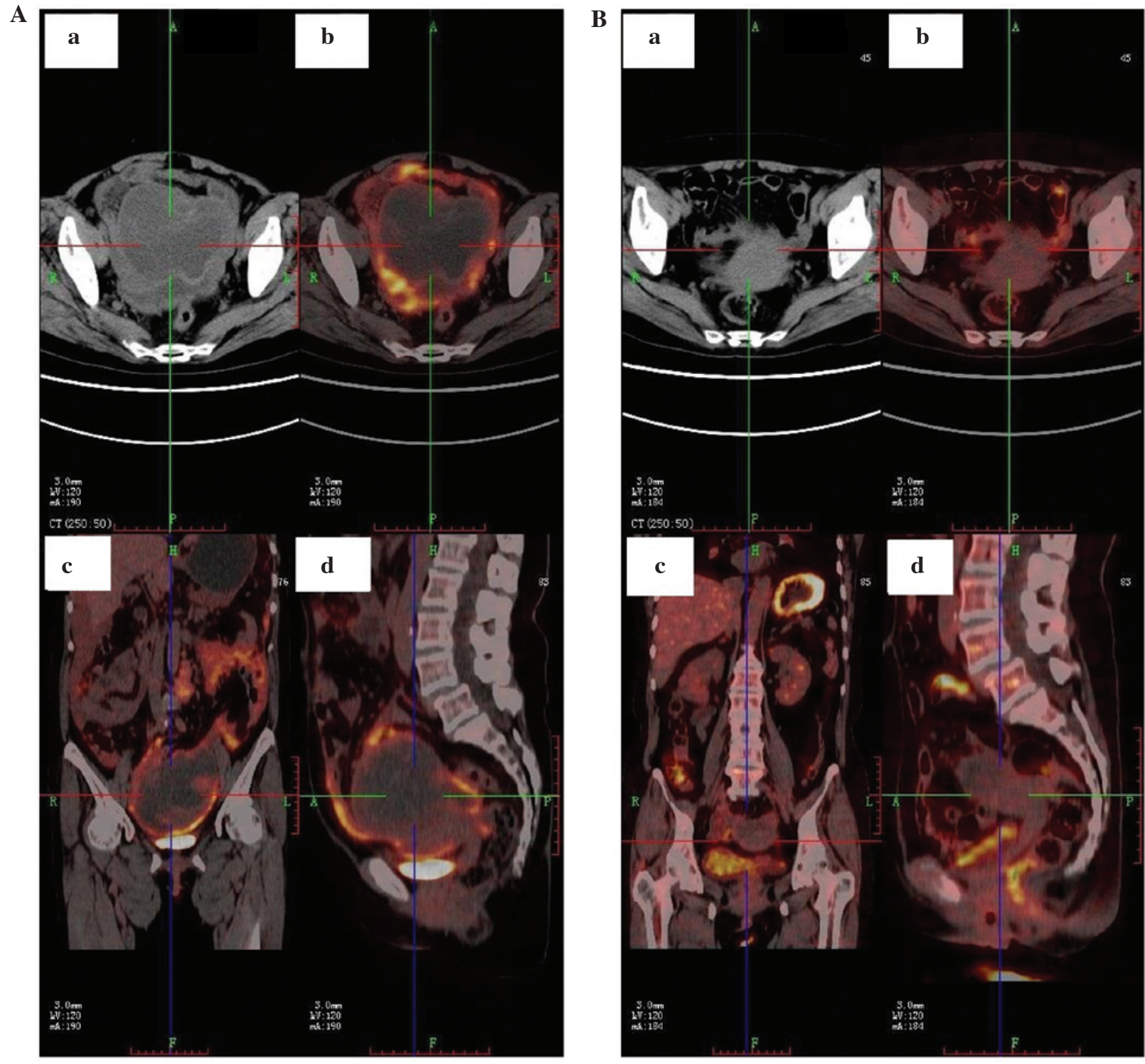

Figure 3. (A) Imaging of the patient prior to treatment. (Aa) Transverse $\mathrm{CT}$ and (Ab) transverse, (Ac) coronary and (Ad) sagittal PET scans of the patient, revealing the presence of metastatic cancer. (B) Imaging of the patient following treatment. (Ba) Transverse CT and (Bb) transeverse, (Bc) coronary and (Bd) sagittal PET scans of the patient, showing the disappearance of the tumor in the pelvic cavity. CT, computed tomography; PET, positron emission tomography.

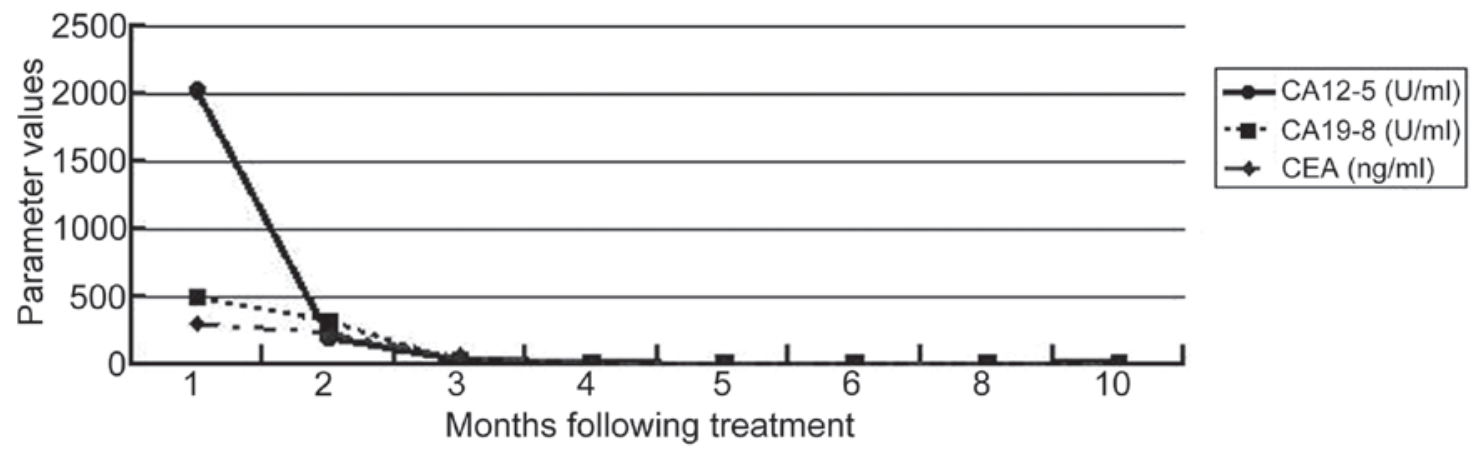

Figure 4. Tumor marker detection. The CA-125 level declined sharply and the CA19-9 and CEA levels declined gradually following radiotherapy treatment. $\mathrm{CA}$, cancer antigen; CEA, carcinoembryonic antigen.

recurrence, such as the diaphragm and liver capsule, and also the pelvic and para-aortic lymph node regions. The treatment effectively spares the kidneys, liver and bone marrow, and is subject to high compliance by patients.
In conclusion, the present study indicates that when adjuvant combination chemotherapy (Taxol and cisplatin) is available, modern radiotherapy techniques, such as BIMRT, may be considered as a beneficial treatment option for ovarian 
cancer patients with multiple peritoneal metastases in whom surgery is not suitable.

\section{References}

1. Bristow RE, Duska LR, Lambrou NC, et al: A model for predicting surgical outcome in patients with advanced ovarian carcinoma using computed tomography. Cancer 89: 1532-1540, 2000.

2. Parazzini F, Valsecchi G, Bolis G, et al: Pelvic and paraortic lymph nodal status in advanced ovarian cancer and survival Gynecol Oncol 74: 7-11, 1999.

3. Pereira A, Magrina JF, Rey V, Cortes M and Magtibay PM: Pelvic and aortic lymph node metastasis in epithelial ovarian cancer. Gynecol Oncol 105: 604-608, 2007.

4. Du Bois A and Pfisterer J: Future options for first-line therapy of advanced ovarian cancer. Int J Gynecol Cancer 15 (Suppl 1): 42-50, 2005.

5. Winter WE III, Maxwell GL, Tian C, et al: Prognostic factors for stage III epithelial ovarian cancer: a Gynecologic Oncology Group Study. J Clin Oncol 25: 3621-3627, 2007.

6. Onda T: Neoadjuvant chemotherapy for ovarian cancer. Gan To Kagaku Ryoho 39: 882-886, 2012.

7. Muggia FM, Braly PS, Brady MF, et al: Phase III randomized study of cisplatin versus paclitaxel versus cisplatin and paclitaxel in patients with suboptimal stage III or IV ovarian cancer: a gynecologic oncology group study. J Clin Oncol 18: 106-115, 2000 .

8. Mouratidou D, Gennatas C, Michalaki V, et al: A phase III randomized study comparing paclitaxel and cisplatin versus cyclophosphamide and cisplatin in patients with advanced ovarian cancer. Anticancer Res 27: 681-685, 2007.

9. Markman M, Bundy BN, Alberts DS, et al: Phase III trial of standard-dose intravenous cisplatin plus paclitaxel versus moderately high-dose carboplatin followed by intravenous paclitaxel and intraperitoneal cisplatin in small-volume stage III ovarian carcinoma: an intergroup study of the Gynecologic Oncology Group, Southwestern Oncology Group, and Eastern Cooperative Oncology Group. J Clin Oncol 4:1001-1007, 2001

10. Kumar A, Gilks CB, Mar C, Santos J and Tinker AV: Patterns of spread of clear cell ovarian cancer: Case report and case series. Gynecol Oncol Rep 6: 25-27, 2013.
11. Petit T, Velten M, d'Hombres A, et al: Long-term survival of 106 stage III ovarian cancer patients with minimal residual disease after second-look laparotomy and consolidation radiotherapy. Gynecologic Oncology 104: 104-108, 2007.

12. Marsden DE, Friedlander M and Hacker NF:Current management of epithelial ovarian carcinoma: a review. Semin Surg Oncol 19: 11-19, 2000.

13. Risum S, Høgdall C, Loft A, et al: The diagnostic value of PET/CT for primary ovarian cancer - a prospective study. Gynecol Oncol 105: 145-149, 2007.

14. FIGO-International Federation of Gynaecologists and Obstetricians Cancer Committee: Annual Report on the Results of Treatment in Gynaecological Cancer. FIGO, Stockholm, 1979

15. Ozols RF, Bundy BN, Greer BE, et al; Gynecologic Oncology Group: Phase III trial of carboplatin and paclitaxel compared with cisplatin and paclitaxel in patients with optimally resected stage III ovarian cancer: a Gynecologic Oncology Group study. J Clin Oncol 21: 3194-3200, 2003.

16. Bristow RE, Tomacruz RS, Armstrong DK, et al: Survival effect of maximal cytoreductive surgery for advanced ovarian carcinoma during the platinum era: a meta-analysis. J Clin Oncol 20: 1248-1259, 2002.

17. Gadducci A, Cosio S, Conte PF and Genazzani AR: Consolidation and maintenance treatments for patients with advanced epithelial ovarian cancer in complete response after first-line chemotherapy: a review of the literature. Critl Rev Oncol Hematol 55: 153-166, 2005.

18. Morrison J, Haldar K, Kehoe S and Lawrie TA: Chemotherapy versus surgery for initial treatment in advanced ovarian epithelial cancer. Cochrane Database Syst Rev 8: CD005343, 2012.

19. Onda $\mathrm{T}$ and Yoshikawa $\mathrm{H}$ : Neoadjuvant chemotherapy for advanced ovarian cancer: overview of outcomes and unanswered questions. Expert Rev Anticancer Ther 11: 1053-1067, 2011.

20. Lambert HE, Gregory WM, Nelstrop AE and Rustin GJ: Long-term survival in 463 women treated with platinum analogs for advanced epithelial carcinoma of the ovary: Life expectancy compared to women of an age-matched normal population. Int J Gynecol Cancer 14: 772-778, 2004.

21. Rochet N, Sterzing F, Jensen AD, Dinkel J, Herfarth KK, Schubert K, et al: Intensity-modulated whole abdominal radiotherapy after surgery and carboplatin/taxane chemotherapy for advanced ovarian cancer: phase I study. Int J Radiat Oncol Biol Phys 76: 1382-1389, 2010 . 